\title{
Impact of mobility in dense LTE-A networks with small cells
}

\author{
B. Baynat*, R-M. Indre**, N. Nya*, P. Olivier**, A. Simonian** \\ *UPMC LIP6,**Orange Labs \\ E-mail: \{bruno.baynat, narcisse.nya\}@lip6.fr, \{ralucamaria.indre, alain.simonian, phil.olivier\}@orange.com
}

\begin{abstract}
In this paper, we investigate the impact of mobility on user performance in the context of dense LTE-A networks. To this end, we propose simple analytical models that capture mobility through the distribution of the mobile users sojourn time, i.e., the time a mobile user is physically present in a given cell. We use these models to derive the throughput of users who remain spatially static during their whole data transmission and the amount of handovers generated by moving users. We analyze the impact on performance of some key parameters such as the size of the small cell, the speed and proportion of mobile users and the distribution of their sojourn time. Numerical evaluation and simulation results are provided to assess the accuracy of the latter model and gain insight into the global system performance.
\end{abstract}

\section{INTRODUCTION}

To cope with the continuous increase in mobile data traffic, network operators envisage massive deployment of small cells so as to enhance the capacity and/or coverage of LTE-A networks [1]. In such dense LTE-A networks, the proportion of mobile users and their impact on system performance is expected to increase. Indeed, moving users will experience various transmission conditions in the overlapping zones between macro-cells and small cells. As a consequence, increased overhead has to be handled due to a higher handover frequency between neighboring cells. In the meantime, static users may benefit from the fact that mobile users traverse the cell. Indeed, the effective load decreases when moving users leave the cell.

Accounting for such complex mobility effects in a simple manner represents an important challenge. As motivated above, this can be formulated as a trade-off between an enhanced performance for static users versus an increase of handovers generated by mobile users. In this context, the present paper addresses the impact of user mobility on the performance of small cells in LTE-A networks. Specifically, we study the influence of mobile users on the performance of static users and the amount of generated handovers.

Although revisited in the present context of LTE-A networks, various user mobility patterns for wireless networks

* This work has been carried out in the framework of IDEFIX project, funded by the ANR under the contract number ANR-13-INFR-0006. have been previously proposed in order to define the relevant modeling characteristics [8]. Two main categories of models can be identified. First, geometric models explicitly represent the random paths of moving users in the plane, e.g., by means of the so-called Random Waypoint (RW) model. In [9], the RW model enables the authors to show that the handover rate is proportional to the square root of the cell density. However, these geometric models do not typically account for the dynamicity of communication traffic (i.e., flow arrivals and departures). On the other hand, non-spatial models address this dynamicity at the expense of a simplified mobility description. Indeed, mobility is simply modeled by assuming that moving users jump between distinct capacity zones according to a Markov process. The capacity gain due to mobility is then estimated through upper and lower bounds associated with fluid limits and quasi-stationary regimes, respectively [3], [4], [5]. Instead of deriving bounds, approximate expressions for the mean flow throughput are derived in [7] by means of a Markovian model for the moving users' displacement.

In this paper, we propose to decompose the mobility problem as follows. We first capture mobility through the distribution of the mobile users sojourn time in a given cell, i.e., the time a mobile user is physically present in the cell. Such a distribution is the output of a chosen mobility model, be it RW, discrete or continuous Markovian, etc. Given this distribution, we then construct a queuing model that allows us to derive performance metrics at the flow level for each user class, namely the mean throughput of static users and the handover probability for mobile users.

\section{NETWORK MODEL}

Consider a macro-cell containing several small cells. Each small cell being of limited range,we consider here that its transmission capacity $C(\mathrm{Mbit} / \mathrm{s})$ is spatially constant, which represents a reasonable assumption for small cells. The case of macrocells with varying capacity will be considered in future work. The macro-cell is visited by both static and mobile users; the latter move inside the macro-cell and may cross one or several small cells before completing a data transmission. We address the impact of these mobile users on 
the performance of users who remain static inside the small cell, and the amount of generated handover. In the following, we interchangeably use the terms "small cell" and "cell".

\section{A. Assumptions and notation}

The transmission capacity $C$ of each small cell is equally shared among all users present in its service area. This can be implemented by means of a Round-Robin discipline and the occupancy of this service system can be modeled by a Processor-Sharing (PS) queue [2]. In other words, at any time $t$, each user is given the instantaneous service rate $C / N(t)$ if the total number of active users in the cell is $N(t)$.

Considering downlink traffic only, we assume that static and mobile users generate requests for transmission in the cell according to independent Poisson processes with rates $\lambda_{s}$ and $\lambda_{m}$, respectively. Note that $\lambda_{m}$ accounts for users that become active inside the considered cell as well as already active users coming from neighbouring cells. Both kinds of requests have an identically distributed volume $\Sigma$ of data to be transferred. As soon as a new request arrives (either from a static or a mobile user), it triggers the start of a new data transmission. For a static user, this transmission lasts until the completion of the whole data transfer; the volume eventually transferred thus equals $\Sigma$. For a mobile user, this transmission can end either because the user has completed its transfer before leaving the cell, or because he has left the cell before completing its transfer. As a result, the volume transferred to a mobile user is always less than (or equal to) $\Sigma$. We denote by $\Theta$ the remaining sojourn time of an active mobile user, that is, the time duration he physically stays in the cell once the transmission has started; note that $\Theta$ can be expressed as $\Theta=D / v$ where $D$ is the random distance the mobile user travels in the cell (from its activation point) and $v$ is the speed of the user, here assumed to be constant. Finally, we denote by $\mu=C / \mathbb{E}(\Sigma)\left(\mathrm{s}^{-1}\right)$ the service rate of the cell, i.e., the departure rate of full transmissions that the cell can handle. A "full transmission" refers either to the transmission of a static user or to that of a mobile user that has not left the cell before completing its transfer. Due to mobility, $\mu$ does not correspond to the actual number of transfers in the cell.

\section{B. Markovian model}

Given the above description, the occupation state of the cell can be described by the bi-dimensional process $\left(N_{s}(t), N_{m}(t)\right)_{t \geq 0}$, where $N_{s}(t)$ (resp. $N_{m}(t)$ ) is the number of static (resp. mobile) ongoing data transfers in the cell at time $t$. The evolution of this process is exactly represented by a so-called Processor-Sharing queue with impatience [6], the "impatient" customers here corresponding to mobile users that may leave the system before their service completion.
To simply characterize the evolution of this PS queue with impatience, we now assume that the volume $\Sigma$ is exponentially distributed with parameter $1 / \mathbb{E}(\Sigma)$ and that the duration $\Theta$ is exponentially distributed with parameter $\theta=1 / \mathbb{E}(\Theta)$, the impatience rate. The process $\left(N_{s}(t), N_{m}(t)\right)_{t \geq 0}$ is then clearly Markovian with transition diagram illustrated in Figure 1; from any state $\left(n_{s}, n_{m}\right), n_{s}>0, n_{m}>0$, we can reach state $\left(n_{s}+1, n_{m}\right)$ with transition rate $\lambda_{s}$, or state $\left(n_{s}-1, n_{m}\right)$ with transition rate $n_{s} \mu /\left(n_{s}+n_{m}\right)$, corresponding to the completion of the transmission of one of the $n_{s}$ static users among a total of $n_{s}+n_{m}$ users. Similarly, we can reach state $\left(n_{s}, n_{m}+1\right)$ with transition rate $\lambda_{m}$, or state $\left(n_{s}, n_{m}-1\right)$ with transition rate $n_{m} \mu /\left(n_{s}+n_{m}\right)+n_{m} \theta$; the second term corresponds to one of the $n_{m}$ mobile users leaving the cell before completing its transfer.

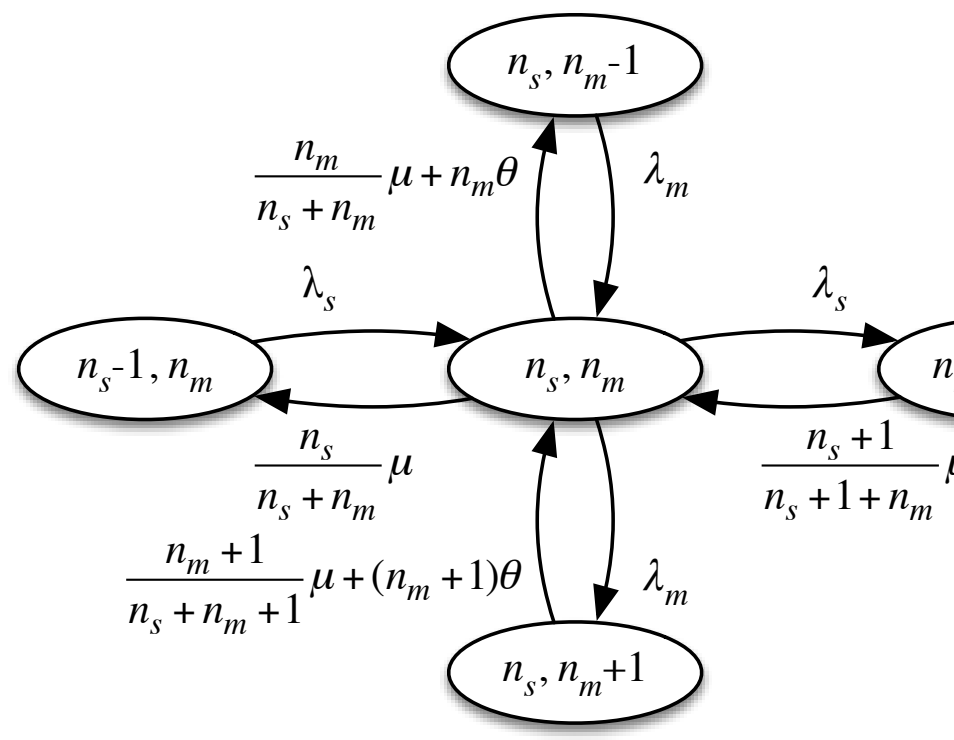

Fig. 1. Transition diagram for the Markov process $\left(N_{s}(t), N_{m}(t)\right)_{t \geq 0}$

By using an appropriate Lyapunov function, it can be simply shown that the occupancy process $\left(N_{s}(t), N_{m}(t)\right)_{t \geq 0}$ has a stationary regime if $\rho_{s}=\lambda_{s} / \mu<1$. Note that this stability condition does not depend on the traffic intensity of mobile (i.e., impatient) users. Intuitively, this can be understood by the fact that the latter always leave the cell after a finite time and therefore cannot cause a system overload. Now fixing $\rho_{s}<1$, it is easily verified from the transition diagram in Figure 1 that the process $\left(N_{s}(t), N_{m}(t)\right)_{t \geq 0}$ is not reversible and thus its stationary distribution $p\left(n_{s}, n_{m}\right), n_{s}, n_{m} \geq 0$, is not amenable to a simple product form. We can nevertheless determine this stationary distribution by solving numerically its associated system of global balance equations, truncating both dimensions of the state space and using any appropriate numerical technique (e.g. Gauss-Seidel or Least mean square). From this distribution, we can derive the performance indicators of interest. First, we calculate the average throughput $\bar{\gamma}$ 
obtained by any static user; by Little's law, $\mathbb{E}\left(N_{s}\right) / \lambda_{s}$ is the mean time to transfer the average volume $\mathbb{E}(\Sigma)$ hence

$$
\bar{\gamma}=\frac{\lambda_{s} \mathbb{E}(\Sigma)}{\mathbb{E}\left(N_{s}\right)} .
$$

We further derive the proportion $H$ of mobile users that exit the cell before the completion of their transmission $(H$ stands for "Handover"), that is,

$$
H=\sum_{n_{s} \geq 0, n_{m} \geq 1} q\left(n_{s}, n_{m}\right) \frac{\left(n_{s}+n_{m}\right) \theta}{\mu+\left(n_{s}+n_{m}\right) \theta},
$$

where $q\left(n_{s}, n_{m}\right)=p\left(n_{s}, n_{m}\right) / \mathbb{P}\left(N_{m} \geq 1\right)$.

\section{A Fixed-Point approximation}

Although the Markovian model can be solved numerically, it does not lend itself to explicit expressions for either the mean throughput $\bar{\gamma}$ or the handover probability $H$. Moreover, it is limited to exponentially distributed variables $\Sigma$ and $\Theta$. We now propose an alternative approximate model that allows deriving such expressions in a wider assumption framework.

Instead of viewing mobile users as impatient customers for the Processor-Sharing queue, we can consider them as belonging to another class similar to that of static users but with a distinct service rate $\mu_{m} \neq \mu$ which accounts for the early departures due to their mobility. Specifically, we represent the cell occupancy by a multi-class ProcessorSharing queue with 2 classes of customers, a static class and a mobile class, as illustrated in Figure 2. As in Section II-A, static (resp. mobile) users still generate requests according to a Poisson process with a rate $\lambda_{s}$ (resp. $\lambda_{m}$ ) and the service rate of static users is $\mu_{s}=\mu=C / \mathbb{E}(\Sigma)$.

Fig. 2. Multi-class PS queue with Fixed-Point approximation

We are now left to estimate the equivalent service rate of mobile users $\mu_{m} \neq \mu$. Let us first assume that the average number of bits $\bar{x}$ transferred by a mobile user before he physically leaves the cell is known. As argued in Section II-A, we have $\bar{x} \leq \mathbb{E}(\Sigma)$; this quantity is obviously related to the total load of the cell and to the physical sojourn time $\Theta$ of mobile users in the cell. From that value of $\bar{x}$, we can write the service rate of mobile users in the PS queue as

$$
\mu_{m}=\frac{C}{\bar{x}} .
$$

Given parameters $\lambda_{s}, \lambda_{m}, \mu_{s}$ and $\mu_{m}$, standard results for the stationary multi-class Processor Sharing queues can be readily applied to calculate the average throughput $\bar{\gamma}$ obtained by both static and mobile users during their transfer, that is,

$$
\bar{\gamma}=C(1-\rho)
$$

where $\rho=\rho_{s}+\rho_{m}$, with

$$
\rho_{s}=\frac{\lambda_{s}}{\mu}=\frac{\lambda_{s} \mathbb{E}(\Sigma)}{C}, \quad \rho_{m}=\frac{\lambda_{m}}{\mu_{m}}=\frac{\lambda_{m} \bar{x}}{C} .
$$

Recall that the stability condition for that multi-class PS queue is $\rho<1$, and it is thus necessary that $\rho_{s}<1$ to ensure the system stability. The sufficiency of condition $\rho_{s}<1$ is less straightforward. However, as argued below, $\bar{x}$ and therefore $\rho_{m}$ will be defined as a function of $\rho_{s}$, say $\rho_{m}=f\left(\rho_{s}\right)$. Based on the numerical evaluations of $\bar{\gamma}$ in Section III-A below, we can conjecture that $\rho_{s}<1$ is sufficient to ensure that $\bar{\gamma}>0$.

We now provide an estimation for the missing parameter $\bar{x}$, required in the expression of $\mu_{m}$. Consider a mobile user with a given volume $\Sigma=y$ to transfer. If he stays in the cell a time $t \geq y / \bar{\gamma}$, he can complete a full transfer. On the other hand, if he stays in the cell a time $t<y / \bar{\gamma}$, this time only enables him to download $\bar{\gamma} t$ bits in average. Given $\Sigma=y$, the mean transferred volume $\bar{x}(y)$ can therefore be expressed as

$$
\bar{x}(y)=\int_{0}^{\frac{y}{\gamma}} \bar{\gamma} t f_{\Theta}(t) \mathrm{d} t+\int_{\frac{y}{\gamma}}^{+\infty} y f_{\Theta}(t) \mathrm{d} t
$$

where $f_{\Theta}$ is the probability density of the time $\Theta$ spent by a mobile user in the cell after the transmission has started. Now, deconditioning expression (6) with respect to the distribution of $\Sigma$ (with probability density $f_{\Sigma}$ ), we obtain

$$
\bar{x}=\int_{0}^{+\infty} \bar{x}(y) f_{\Sigma}(y) \mathrm{d} y
$$

with $\bar{x}(y)$ expressed in (6). We thus end up with a system of four dependent equations (3)-(4)-(5)-(7) that provide a FixedPoint equation for unknown $\mu_{m}$, or equivalently $\bar{\gamma}$. Once $\bar{\gamma}$ is determined by the Fixed-Point equation, the handover probability $H$ can then be estimated in turn by

$$
H=\int_{0}^{+\infty} \mathbb{P}\left[\Theta \leq \frac{y}{\bar{\gamma}}\right] f_{\Sigma}(y) \mathrm{d} y .
$$

Despite its simplicity, the above Fixed-Point approach is, however, an approximation in that we implicitly assume in both relations (7) and (8) that a mobile user always receives an average throughput $\bar{\gamma}$ along its entire sojourn in the cell.

If we assume, as previously, that $\Sigma$ and $\Theta$ are exponentially distributed; integral (7) then readily gives

$$
\bar{x}=\frac{\sigma \bar{\gamma}}{\sigma \theta+\bar{\gamma}},
$$

where $\sigma=\mathbb{E}(\Sigma)$ and $\theta=1 / \mathbb{E}(\Theta)$. This relation enables us to easily reduce the latter set of equations (3)-(4)-(5)-(7) to a simple quadratic equation in $\bar{\gamma}$ with a unique positive solution, thus providing the average throughput

$$
\bar{\gamma}=\frac{C-\sigma\left(\lambda_{s}+\lambda_{m}+\theta\right)+\sqrt{\Delta}}{2},
$$

where $\Delta=\left(C-\sigma\left(\lambda_{s}+\lambda_{m}+\theta\right)\right)^{2}+4 \theta \sigma\left(C-\lambda_{s} \sigma\right)$. Finally, applying (8) to exponentially distributed $\Sigma$ and $\Theta$ gives

$$
H=\frac{\sigma \theta}{\sigma \theta+\bar{\gamma}} .
$$


Note however that, unlike the first Markovian model, this second PS queue model can account for wider range of assumptions for the distributions of both the sojourn time $\Theta$ of a mobile user in the cell and the volume $\Sigma$ of data a user has to transfer, while staying very easy to implement.

\section{Performance Results}

We have proposed two models that account for the mutual impact of static and mobile users in a radio cell, namely a Markovian model and a Fixed-Point approximation. As long as the assumptions of Section II-B are satisfied, the Markovian model is exact. However, if one of the above-mentioned assumptions is not satisfied anymore (e.g., one of the random variables is no longer exponentially distributed), exact results can no longer be derived using the Markovian model and we need to resort to simulation to validate both models.

\section{A. Model validation}

We first compare the results provided by the two models in the case where $\Theta$ and $\Sigma$ are both exponentially distributed. We set $C=40 \mathrm{Mbit} / \mathrm{s}$ for the cell capacity and $\sigma=10$ MB for the mean flow volume. The mobile users speed is set to $v=50 \mathrm{~km} / \mathrm{h}$ and the mean distance crossed by mobile users is $\mathbb{E}(D)=100 \mathrm{~m}$, yielding a mean exit rate $\theta=$ $v / \mathbb{E}(D)=0.14 \mathrm{~s}^{-1}$. Figure 3 compares the mean throughput of static users $\bar{\gamma}$ obtained by the two models, in terms of the load $\rho_{s}$ induced by static users. The continuous (resp. dotted) lines correspond to the results of the exact Markovian model (resp. the Fixed-Point approximation). Each of the three sets of curves corresponds to a proportion of mobile users $p \in\{0.2,0.5,0.8\}$. We observe that the results provided by the Fixed-Point approximation match the exact results with a good accuracy (with a relative error less than $10 \%$ in most cases), and provide a lower bound (i.e., conservative) for the static user throughput in all cases. From these results we note, as expected, that the throughput of static users degrades considerably as the proportion of mobile users increases, thus increasing the total load $\rho=\rho_{s}+\rho_{m}$.

Figure 4 represents the handover probability $H$ of mobile users, still as a function of the load $\rho_{s}$ of static users. We use the same numerical values for all parameters, but for sake of clarity we just represent two sets of curves corresponding to a proportion of mobile users $p \in\{0.2,0.5\}$. When the load of static users is very low, i.e., $\rho_{s} \rightarrow 0$, then $\bar{\gamma} \rightarrow C$ and in view of (11), we have $H \rightarrow \sigma \theta /(\sigma \theta+C)$; with the considered parameters this yields $H \rightarrow 0.22$ when $\rho_{s} \rightarrow 0$. The handover probability then increases from this initial value as the load of the static users increases and $H$ tends to 1 when $\rho_{s}$ approaches 1 . Note that the Fixed-Point approximation is fairly accurate at relatively low loads and becomes slightly less accurate as the load increases.

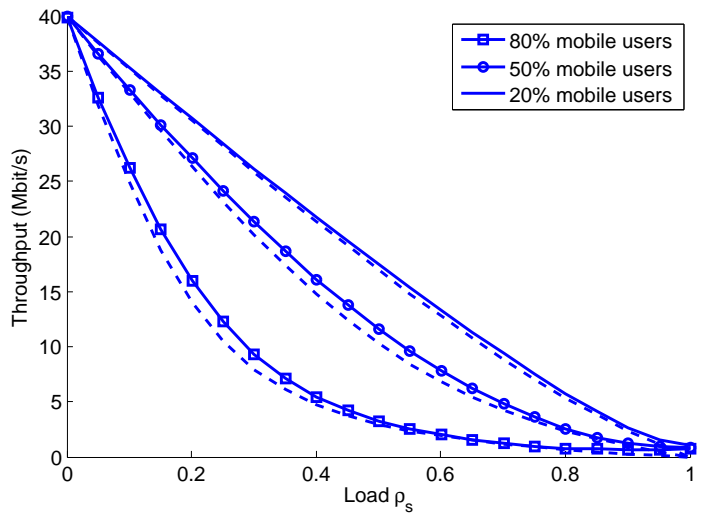

Fig. 3. Static user throughput as a function of load $\rho_{s}$ for different proportions of mobile users ( $v=50 \mathrm{~km} / \mathrm{h}$ and mean crossed distance $\mathbb{E}(D)=100 \mathrm{~m}$ )

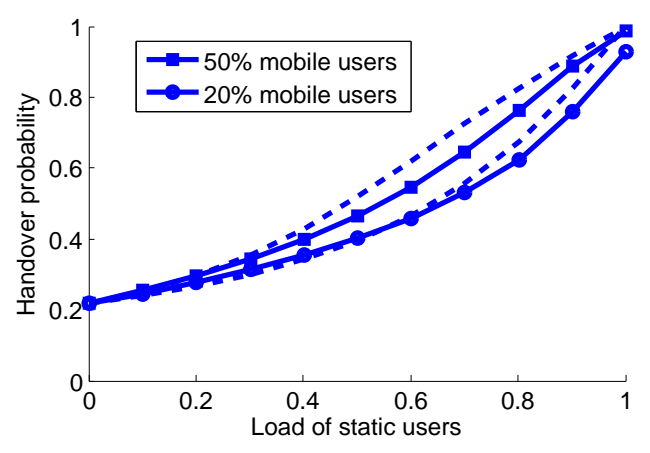

Fig. 4. Handover probability as a function of load $\rho_{s}$

We now evaluate the robustness of models with regard to the distribution of the sojourn time $\Theta$ of mobile users in the cell. The exponential distribution previously considered for $\Theta$ is probably not a realistic assumption. Indeed, if we consider a mobile user moving in the cell along a diameter at constant speed and whose initial position is uniformly distributed on that diameter, it can be easily shown in this case that $\Theta$ is uniformly distributed. Figure 5 thus depicts the throughput of the static users as a function of the static user load for different distributions of the mobile user sojourn time. Values for the uniform and deterministic distribution are obtained through simple event-driven flow-level simulations, while those for the exponential distribution are obtained by numerically solving the Markovian model. We note that the throughput performance is only marginally impacted by the sojourn time distribution indicating that derived results remain valid for more realistic sojourn time distributions.

\section{B. Impact of key parameters}

We now use the Fixed-Point approximation to discuss the impact of some key system parameters such as the speed of mobile users or the cell size. Figure 6 depicts the variations of $\bar{\gamma}$ with respect to the cell crossing speed $v$ for different values 


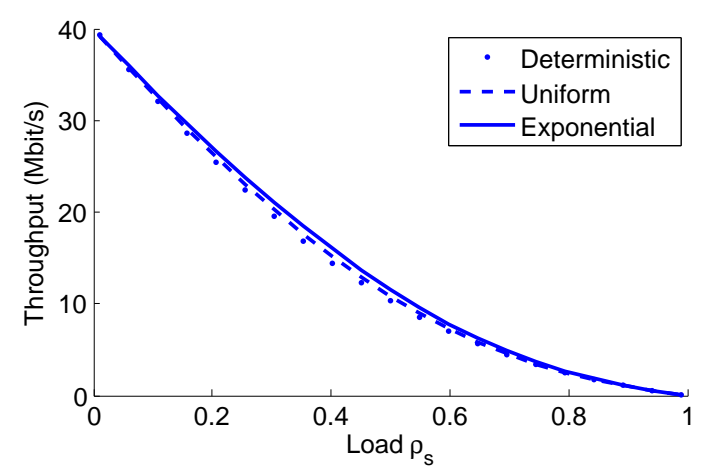

Fig. 5. Static user throughput as a function of load $\rho_{s}$ for different distributions of mobile users' sojourn duration

of the static load $\rho_{s} \in\{0.2,0.5,0.8\}$ (from top to bottom) and a fixed proportion of mobile users $p=0.5$. Note from (4) that the bounds $\bar{\gamma}(0) \leq \bar{\gamma} \leq \bar{\gamma}(+\infty)$ hold, where $\bar{\gamma}(0)=C(1-\rho)$ with $\rho=\left(\lambda_{s}+\lambda_{m}\right) \sigma / C$ corresponds to the case when all users are static $(\theta=0)$, and $\bar{\gamma}(+\infty)=C\left(1-\rho_{s}\right)$ corresponds to mobile users with infinite speed $(\theta=+\infty)$ and a vanishing impact on static users. The variations of the throughput in terms of $\theta$ are therefore significant for relatively small values of $\rho_{s}$. Note also that, as expected, the throughput $\bar{\gamma}$ of static users increases when the speed $v$ increases, validating the fact that static users benefit from increased mobility.

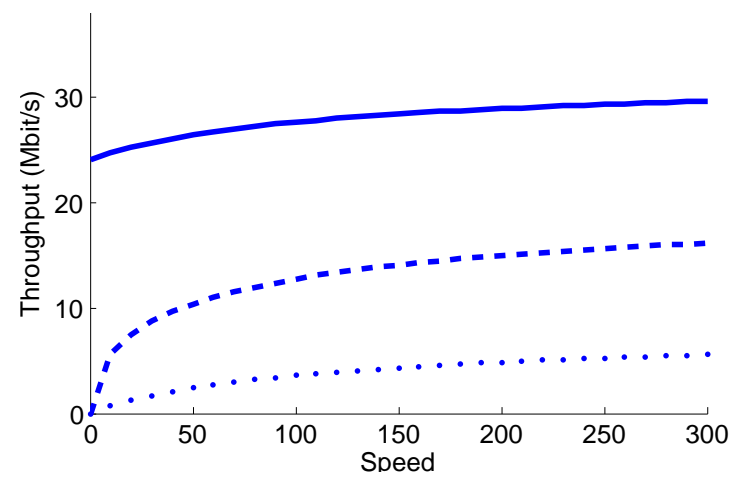

Fig. 6. Static user throughput as a function of mobile users' speed for different loads $\rho_{s} \in\{0.2,0.5,0.8\}$ (from top to bottom)

In Figure 7, we assess the impact of the cell size by considering a typical Micro, Pico or Femto cell with a diameter of $1 \mathrm{~km}, 100 \mathrm{~m}$ or $10 \mathrm{~m}$, respectively; we also consider that the mean sojourn durations are proportional to these values ( $v$ is fixed to $5 \mathrm{~km} / \mathrm{h}$, e.g. typical of a pedestrian). As expected, users in Femto cells experience the highest throughput since they benefit from the least interaction with the mobile users.

\section{CONCLUSION}

We have analyzed the impact of mobility on user performance in LTE-A networks with small cells. Two simple

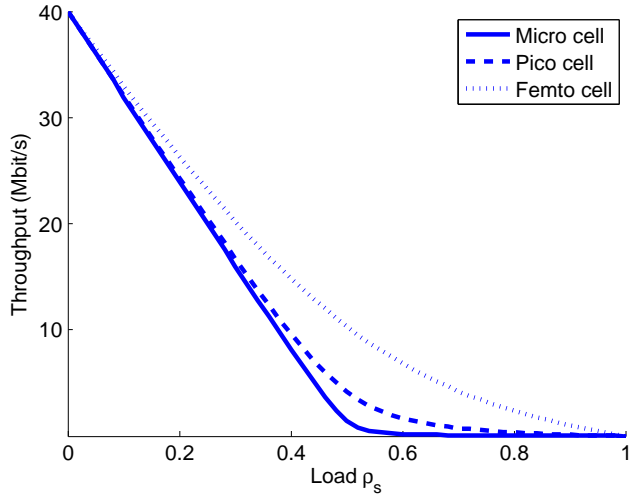

Fig. 7. Static user througput as a function of load $\rho_{s}$ for different cell sizes

analytical models have been proposed that capture mobility through the distribution of the sojourn time of mobile users: a Markovian model and a Fixed-Point approximation model. The first one is exact as long as all distributions involved in the modeling process are assumed to be exponential, but relies on a bi-dimensional Markov process that has to be solved numerically. The second one can manage a wider range of assumptions for the distributions of variables and is very easy to implement, but remains an approximation even when all distributions are exponential. In cases where the first model is exact, we have shown that the second one is able to predict user performance with a good accuracy. Based on the proposed models, we were able to quantify the impact of key mobility parameters such as speed, proportion of mobile users and cell size. By means of simulations, we have shown that the derived results are robust with respect to different distributions of the mobile users' sojourn time.

\section{REFERENCES}

[1] 3GPP TS 25.214 version 10.2.0, Physical layer procedures (FDD), release 10

[2] T.Bonald, A.Proutière, Wireless downlink data channels: user performance and cell dimensioning, MobilCom'03, Sept.2003, San Diego, USA

[3] T.Bonald, S.Borst, N.Hegde, How mobility impacts the flow-level performance of wireless data systems, IEEE Infocom 2004

[4] T.Bonald, S.Borst, N.Hegde, M.Jonckheere, A.Proutière, Flow-level performance and capacity of wireless networks with user mobility, Queueing Systems, 63:pp.131-164, 2009

[5] S.Borst, A.Proutière, N.Hegde, Capacity of wireless data networks with intra- and inter- cell mobility, IEEE Infocom 2006

[6] E.G.Coffman, A.A.Puhalskii, M.I.Reiman, P.E.Wright, Processor-shared buffers with reneging, Performance Evaluation, vol.19, pp.25-46, 1994

[7] M.K.Karray, User's mobility effect on the performance of wireless cellular networks serving elastic traffic, Wireless Networks, 17, pp.247-262, 2011

[8] C.Schindelhauer, Mobility in wireless networks, SOFSEM, J.Wiedermann et al. ed., pp.100-116, 2006

[9] X.Lin, R.K.Ganti, P.J.Fleming, J.G.Andrews, Towards understanding the fundamentals of mobility in cellular networks, IEEE Transactions on Wireless Communications, vol.12, No.4, April 2013 